\title{
El hipermedia móvil como nuevo medio y su protagonismo en la creación transmedia*
}

\section{Resumen}

Mobile First no es solo un concepto fruto de la mercadotecnia, pues hasta la UNESCO invita a que se exploren las posibilidades educativas del móvil o celular dentro y fuera del aula. Internet móvil, internet de las cosas, geolocalización y sensores de dispositivos móviles configuran una nueva convergencia de medios marcada por la movilidad de persona y aparato. Esta movilidad, además de interactividades (relaciones con intermediación tecnológica), característica de los sistemas estáticos, genera relaciones analógicas debido a las interacciones (relaciones físicas y personales) con el propio usuario, con otras personas y con el binomio espacio-tiempo. Esta movilidad es protagonista en los procesos de diseño y creación transmedia. Diseñadores, creadores y desarrolladores de contenidos y de software tienen que pensar primeramente en este dispositivo (Mobile Firts) y en que sus creaciones se adapten al resto de pantallas (Responsive Web Design). La hipótesis es que el hipermedia móvil se está configurando como un nuevo medio que suele ser el protagonista principal en la creación transmedia. Se plantea así una aproximación taxonómica, narrativa, creativa y ludoeducomunicativa que intenta demostrarlo. Se analizan las posibilidades hipermedia de la comunicación móvil y sus roles en la comunicación transmedia. Se recurre a la prospectiva para señalar las carencias del momento y apuntar posibilidades futuras que nacen de necesidades no cubiertas. El marketing se encarga de potenciar el deslumbramiento tecnológico. Desde la academia es necesaria una mirada rigurosa centrada en el diseño, en los contenidos y en la accesibilidad de manera que ayude a pasar de una tecnología que deslumbra a una tecnología que alumbre el conocimiento.

* Artículo resultante de la investigación "El hipermedia móvil y sus aplicaciones hipermedia y transmedia: investigación, creación y accesibilidad" financiado por la Pontificia

Universidad Javeriana de Cali y la Universidad Complutense de Madrid. Grupos de investigación Museum I+D+C de la Universidad Complutense de Madrid y Destino de la Pontificia Universidad Javeriana de Cali.
PhD. Isidro Moreno Sánchez

Profesor titular de la Universidad Complutense de Madrid, España Grupo de investigación Museum $\mathrm{I}+\mathrm{D}+\mathrm{C}$

Correo: ims@ucm.es

๑ orcid.org/0000-0001-5820-8523

PhD. Andrés Adolfo Navarro

Newball

Profesor asociado de la

Pontificia Universidad Javeriana de Cali, Colombia

Grupos de investigación Destino y Museum I+D+C

Correo: anavarro@javerianacali. edu.co

ำ /orcid.org/0000-0002-4231-8661

Recibido: marzo 2016 Aprobado: abril 2016

Palabras clave:

Accesibilidad, hipermedia móvil, interacción, interactividad, transmedia.

Revista KEPES Año 13 No. 13 enero-junio 2016, págs. 145-170 ISSN: 1794-7111(Impreso) ISSN: 2462-8115 (En línea) DOI: $10.17151 /$ kepes.2016.13.13.8 


\section{Mobile hypermedia as new media and its role in transmedia creation}

\begin{abstract}
Mobile First is not just a concept resulting from marketing since even UNESCO invites to explore the educational possibilities of the mobile phone inside and outside the classroom. Mobile Internet, internet of things, geolocation and mobile sensors configure a new convergence of media characterized by the mobility of people and machines. This mobility, besides making possible interactivities (relations with technological intermediation) characteristic of static systems, generates analogue relationships because of interactions (physical and personal relationships) with the user, with others and within the space-time binomial. This mobility is also the protagonist in the processes of transmedia design and creation. Designers, creators and content and software developers have to think first of this device (Mobile First) and have to consider if its creations fit other screens ("Responsive Web Design). The hypothesis is that mobile hypermedia is emerging as a new media that is usually the main protagonist in transmedia creation. This raises a taxonomic, narrative, creative and ludic-educative-communicative approach that tries to demonstrate it. Hypermedia mobile communication possibilities are analyzed and their roles in transmedia communication. Prospective is used to point out the shortcomings of the moment and to make a note of future possibilities that are born from unmet needs. Marketing is responsible for enhancing the technological glare. A rigorous look from the academy focused on design, contents and accessibility is required so that it helps to go from a dazzling technology to a technology that enlightens knowledge.
\end{abstract}

Key words:

Accessibility, mobile hypermedia, interaction, interactivity, transmedia. 


\section{Introducción}

Ya en 1979, la UNESCO reconocía la importancia de la educación en materia de comunicación, un campo inter y transdisciplinar que permite un conocimiento de los medios y facilita una mirada crítica sobre los mismos. En los bolsillos de la mayoría de las personas existe un potente dispositivo (smartphone) que facilita la apropiación social del arte, la ciencia y la tecnología tanto en educación formal como en procesos creativos y ludo-educomunicativos, gracias a sus posibilidades hipermedia o multimedia interactivas, que lo están configurando como un nuevo medio (Figura 1. London Street Museum. Picadilly Circus en 1953 y hoy: http://goo.gl/0Nuf7m). Una forma de acercarse a estas nuevas creaciones es practicarlas a través del hipermedia móvil comprendiendo su especificidad y utilizándolo de una manera activa construyendo propuestas, propuestas que adquieren un protagonismo cada vez mayor en los procesos transmedia. En ese caso, partiendo de los mismos contenidos, se desarrollan éstos utilizando todo tipo de medios tradicionales y aquellos basados en las tecnologías de la información y la comunicación teniendo muy presente que las personas son parte activa de la creación hasta convertirse en coautores o lectoautores. Ese protagonismo genera, por ejemplo, el fenómeno Fan Fiction. El más destacado es el referido a la cinematografía, pero puede generarse con cualquier creación. De ahí que un objetivo clave de la transmedialidad sea incentivar la participación constructiva. El celular es una potente herramienta para la creación de cualquier tipo y también para la apropiación del territorio, comenzando, por ejemplo, con el trabajo de campo (Gómez, 2015, p. 132). En este caso, el autor o autores pueden solicitar la cocreación colaborativa para generar un nuevo proyecto. 


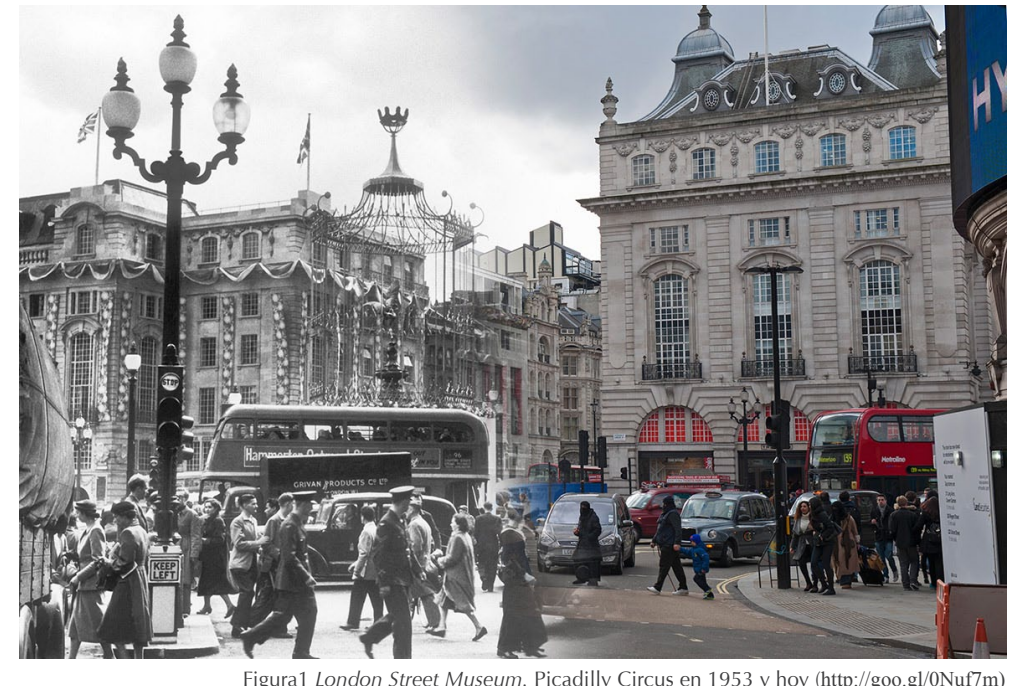

Se es consciente de que la cocreación demanda una investigación específica; aún así, para destacar su importancia, se apuntan algunas direcciones de la misma. A veces, como en el mencionado Fan Fiction, el autor no necesariamente busca la coautoría y, de hecho, las cocreaciones que realizan los fans enriquecen el proyecto transmedialmente, pero no cambian la obra original. En otros casos, el autor, valiéndose de las TIC, puede buscar esa participación y facilitar la transformación directa del propio original con las propuestas de los lectoautores o reservarse esa transformación redefiniendo o eligiendo qué propuestas incluye él mismo de las sugeridas. Estas participaciones transformativas y constructivas de los receptores mutados en coautores marcan la madurez de los hipermedia, ya que en los comienzos la tecnología permitía exclusivamente la participación selectiva. 
En 1979, Andrew Lippman y Robert Mohl, con el apoyo de otros investigadores del MIT Medialab, crearon Aspen Moviemap, el primer sistema de vídeo interactivo aprovechando las posibilidades tecnológicas del recién inventado videodisco o laser disc y de las primeras pantallas táctiles. Más que vídeo interactivo, era un sistema de fotografías interactivas que producían la ilusión de viajar por las calles de la ciudad. Aquellas complejas plataformas lectoras con ordenador, pantalla táctil y lectores de videodisco alumbraron un nuevo medio (hipermedia o multimedia interactivos) que definiría el último cuarto del siglo XX y continúa con un protagonismo aún mayor en el primero del siglo XXI.

Con la simplificación de las plataformas de lectura, se materializaba el "tal como pensamos" de Vannevar Bush (1945) y se despejaba la vía al "tal como hacemos" (el propio cuerpo actúa como interfaz comunicándose con el sistema interactivo sin elementos intermediadores). La multiconvergencia de medios y arquitecturas tecnológicas en cualquier pantalla es ya una realidad. Las pantallas móviles han ido creciendo en potencia hasta llegar a los smartphones o teléfonos inteligentes. Este calificativo mercadotécnico refleja exclusivamente que el aparato incorpora un ordenador. Es el mismo caso de la smart TV. La computación móvil acaba con el protagonismo de los sistemas fijos que han predominado durante 50 años (Townsend, 2006).

Casi cualquier dispositivo móvil (smartphones, tablets, phablets, wearables...) puede soportar sistemas multimedia interactivos. Esta evolución hacia la movilidad ha dotado a estas pantallas de características técnicas singulares como los acelerómetros o el GPS, susceptibles de utilizarse expresivamente. Los autores, como hicieran Lippman y Mohl con las plataformas de videodisco, han ido imaginando productos interactivos aprovechando estas singularidades tecnológicas dando lugar a los locative media o medios geolocalizados que presentan características adecuadas para múltiples tipos de producción orientada a la creación pura y a la ludo-educomunicativa. 
La realidad aumentada en movilidad permite, por ejemplo, descubrir las distintas capas históricas que han existido en un lugar significativo de una ciudad o explorar las obras de ingeniería que esconde el asfalto, los restos arqueológicos o profundizar más allá del subsuelo modificado por las personas para adentrarse en los estratos geológicos. University of South Australia denomina Magic Vision al laboratorio que desarrolla estas propuestas. La misma universidad ha creado, además, el Wearable Computer Laboratory para profundizar en los dispositivos vestibles.

El walking documentary, el walking cinema, el walking audio... sacan los medios audiovisuales al exterior para que interaccionen in situ con el espacio natural o con el espacio urbano de manera que realidad y virtualidad se completen y complementen potenciando la experiencia participativa completa, algo fundamental para la apropiación y la creación de conocimiento a través de la coautoría.

La pantalla con internet móvil que facilita este nuevo medio es la más completa y compleja de las existentes. La estrategia Mobile First + Responsive Web Design recomienda pensar primero en el diseño y los contenidos para el móvil y pensarlos de forma que se adapten después al resto de pantallas. Y esto es así porque, según distinto informes como Ditrendia (2015), ya en ese año, por primera vez, el número de celulares superó al de personas en el mundo y se vendieron 1245 millones de smartphones. También por primera vez, el promedio de tiempo que las personas pasaron frente al televisor (168 minutos) fue inferior al que dedicaron al celular (177 minutos). Europa del Este es la región que tiene una mayor penetración de celulares (139\%), seguida de América del Sur (126\%). En España, el 87\% de los celulares son smartphones, liderando así el mercado europeo de estos dispositivos. En 2016 la mitad de la población mundial tiene ya un smartphone. La academia tiene que ser sensible a esta realidad y aprovechar sus posibilidades expresivas y participativas. 


\section{Objetivos, hipótesis y metodología}

Aproximarse a los rasgos diferenciadores de los multimedia interactivos o hipermedia que facilita internet móvil, a su accesibilidad (física, económica, cognitiva, cultural, tecnológica y de contenidos) y a su narrativa para vislumbrar sus posibilidades creativas y ludo-educomunicativas presentes, apuntando algunas de las futuras, son los objetivos principales de esta investigación. Y todo ello enfocado a los procesos de aprendizaje y a la potenciación de nuevas investigaciones básicas y aplicadas sobre el hipermedia móvil y su protagonismo en los procesos transmedia.

Se parte de la hipótesis de que internet móvil propicia nuevas formas de multimedia interactivos o hipermedia con características diferenciadas que adquieren autonomía respecto al medio del que derivan hasta convertirse en un nuevo medio: el hipermedia móvil. El hipermedia resulta de una convergencia interactiva de medios existentes; el hipermedia móvil añade a esa convergencia la movilidad, y dicha movilidad conlleva también convergencias analógicas e interacciones con las personas, el entorno y con el propio dispositivo, algo fundamental para la comunicación y la educación.

La investigación nace con un objetivo claro de interdisciplinariedad y utiliza el análisis de contenido para sistematizar las características diferenciadoras del hipermedia móvil e intentar demostrar que son constitutivas de un nuevo medio. La importancia cuantitativa de internet móvil, como se señala en la introducción, queda demostrada en todos los estudios nacionales e internacionales, como el citado Ditrendia (2015). Las cifras apuntadas hablan del potencial del hipermedia móvil. 
Desde el punto de vista cualitativo, se han investigado:

- Las diferencias provenientes del uso del dispositivo respecto a los aparatos estacionarios a través de las interfaces de hardware y software.

- La interactividad y la interacción.

- Las relaciones narrativas con el usuario como personaje, así como con el espacio y el tiempo para la generación de acciones.

- Los géneros específicos de este nuevo medio y sus aplicaciones.

- Autoría y coautoría.

- Convergencias con otras pantallas y grado de importancia de la pantalla móvil.

- Su accesibilidad física, cognitiva, cultural, económica, tecnológica y de contenidos

Los grupos implicados en la investigación están especializados en comunicación hipermedia y transmedia e ingeniería de sistemas orientada a videojuegos y computación gráfica. El trabajo realizado es interdisciplinar gracias a que ambos grupos ya han desarrollado anteriores investigaciones conjuntas implicándose en todo el proceso, como la investigación I+D+i Conocimiento aumentado y accesibilidad. La representación museográfica de contenidos culturales complejos (Moreno y Navarro, 2013). Se busca huir de la multidisciplinariedad en la que cada investigador proporciona una parte del puzzle sin implicarse en el resto, dejando así unas heridas científicas evidentes en el conjunto. Landow (2006, p. 1), al repasar binomios de autores de la teoría cultural y de la tecnología, lo refleja así: "Los que trabajan en el campo de los ordenadores conocerán bien las ideas de Nelson y Van Dam; y los que se dedican a la teoría cultural estarán familiarizados con las ideas de Derrida y de Barthes". Destaca la importancia de conocer ambos universos. Porque no se está hablando de prótesis tecnológicas que potencien la incomunicación, sino de dispositivos que faciliten unas mejores relaciones con el entorno y con las personas. 
El cine creó la ilusión de movimiento mediante la convergencia de la linterna mágica, la fotografía y, posteriormente, la fonografía de una manera lineal; los Hipermedia realizan una convergencia interactiva de medios audiovisuales, fotográficos, infográficos y tipográficos, y el hipermedia móvil añade a las convergencias hipermedia una doble movilidad: la movilidad del dispositivo en cualquier tipo de situación aunque la persona no esté en movimiento, y la movilidad de persona y dispositivo (Figura 2. Goya en el Madrid digital. La ermita del Santo en tiempos de Goya y hoy. Grupo Museum I+D+C y Museo del Prado). Añade, además, convergencias analógicas mediante interacciones del dispositivo con otras personas y con el entorno. Son precisamente estas interacciones el principal elemento diferenciador del medio.

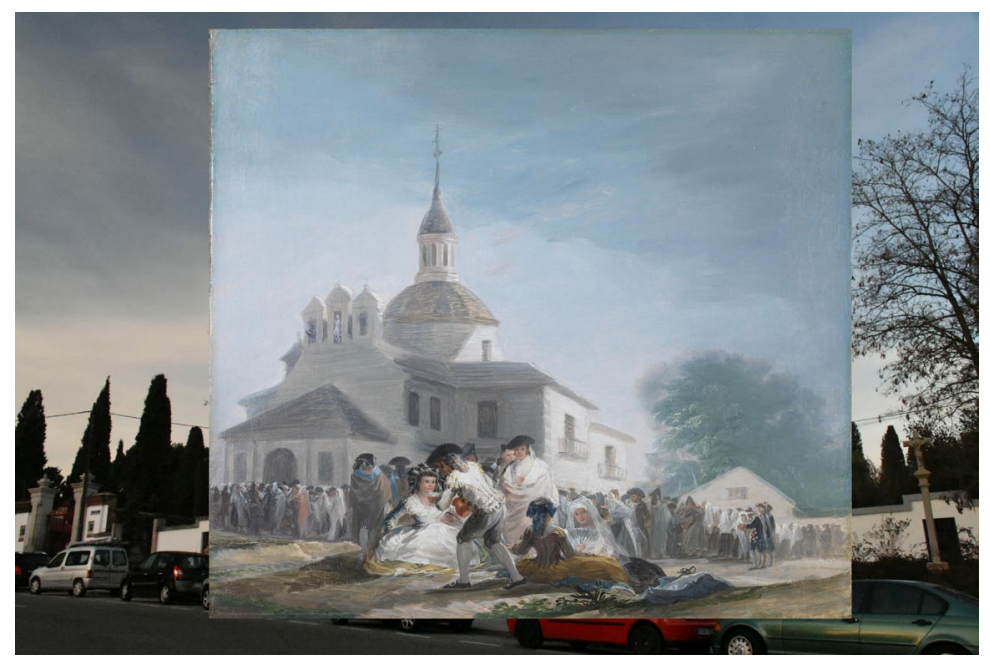

Figura 2. Goya en el Madrid digital. La ermita del Santo en tiempos de Goya y hoy. Grupo Museum I+D+C y Museo del Prado 
Aunque se suelen utilizar como sinónimos, interactividad, como ya se ha apuntado, conlleva intermediación tecnológica, mientras que interacción (Moreno, 2015, pp. 89-90) se aplica a relaciones físicas e interpersonales. Teniendo en cuenta esta diferencia, aparecen algunas de las características definitorias de este nuevo medio que sistematiza la investigación:

\section{Interfaces mimético-naturales e inmersión}

La interfaz, además de la tactilidad, añade el movimiento para acercarse a la participación mimético-natural, al "tal como hacemos". Si la utilización del tacto supone una cierta interacción mimético-natural, el movimiento del aparato para seleccionar o jugar supone una mayor apropiación de las acciones que se desencadenan y un aumento de la libertad interactiva. Este cambio es tan evidente que le llevó a Rico (2010) a evaluar la aceptación social de estos gestos. Gestos que potencian la inmersión o sensación de introducirse en un ambiente determinado, ya que el grado de inmersión está directamente relacionado con los sentidos (inmersión física) y con las emociones (inmersión psicológica). El mayor grado de inmersión física alcanzable con los dispositivos móviles conlleva también el máximo aislamiento; por ejemplo, utilizando el celular con unas gafas especiales y unos auriculares para adentrarse en una aplicación de realidad virtual. Al unir interactividad (inmersión tecnológica) e interacción (inmersión analógica) y potenciando la imbricación de sensaciones (inmersión física) y emociones (inmersión psicológica) a través del relato participativo, se puede lograr un cierto grado de inmersión sin aislamiento.

\section{Interactividad con interacción}

La movilidad propicia la interactividad con interacción. Interacción interfacial física, interactividad con la máquina e interacción con las personas que manejan otros dispositivos fijos o móviles. Se elimina la constricción temporal 
que conlleva tener la máquina anclada a un espacio fijo al que hay que acudir para comunicarse. Esta facilidad de comunicación continua no está libre de excesos y pueden generarse conductas adictivas (Beranuy, Chamarro et. al., 2009). Destacan los autores que el uso problemático de las tecnologías móviles se relaciona con la adolescencia. Ahí es precisamente donde hay que potenciar las aplicaciones educativas. Con la edad, según los autores, se tiende a un uso más profesional y menos lúdico de los dispositivos móviles, produciéndose menos consecuencias negativas.

Convine recordar, que, el móvil o celular alcanza el máximo nivel de interactividad atendiendo al hardware. Si se repasan estos niveles, el nivel de interactividad 0 se corresponde con los sistemas lineales, como el cine visto en una sala tradicional. El nivel 1 se aplicaba a los antiguos magnetoscopios videográficos en los que se podía pausar, adelantar, retrasar... el programa. Con el nivel 2 se designan aquellos equipos, como los primeros lectores de DVD, que propician una interactividad muy sencilla por capítulos. El nivel 3 se corresponde con la interactividad que facilita una computadora. Al conectar esa computadora a internet se alcanza el nivel 4. Si la computadora conectada a internet puede usarse en movilidad interactuando e interaccionando con el espacio y el tiempo (celular), se está en el máximo nivel actual, el número 5.

\section{Nuevos géneros e interacciones con personas y entorno}

Cuando persona y aparato están en movilidad, el hipermedia móvil presenta las características que lo diferencian y personalizan para poder considerarlo un nuevo medio. El hecho de que la persona se desplace es susceptible de generar interacciones que se aprovechan para crear aplicaciones múltiples orientadas a la creación, a la educación, a la publicidad, a los videojuegos o a completar y complementar procesos transmedia. Walking cinema (vídeo geolocalizado), walking documentary (documental geolocalizado), walking audio (audio geolocalizado)... son ejemplos de nuevos géneros asociados exclusivamente 
al hipermedia en movilidad. No hay que olvidar que los usos cotidianos conversacionales más sencillos con los dispositivos móviles también generan narrativas multimedia con interactividad e interacción. Narrativas en movilidad que, según Grassi, Gagglioli y Riva (2009), al propiciar una comunicación multimedia interpersonal sin que espacio y tiempo tengan que coincidir, pueden reducir incluso el estrés, por ejemplo de los commuters, concepto anglosajón para designar a las personas que realizan un viaje considerable todos los días desde el hogar al trabajo.

\section{Lectoautoría y autoría}

En los hipermedia, como se ha dicho, el lector se convierte en lectoautor o coautor en distintos grados, ya que puede seleccionar, transformar e, incluso, construir. Aunque los sistemas de sobremesa permiten todos estos grados de participación, con la movilidad se acentúan, por ejemplo, en el campo de la creación fotográfica y audiovisual. Afirma Calvi (2010, p. 46) que el lenguaje audiovisual se constituye como una de las formas de expresión simbólica más extendida en términos económicos y culturales en movilidad.

\section{Convergencia de pantallas y polivalencia de usos}

Para estas pantallas móviles existen aplicaciones que permiten su convergencia con las pantallas fijas de los ordenadores, de la smart TV y de otras especiales. Es posible compartir pantalla para ver los contenidos ampliados e intercambiarlos con otras personas, incorporar el celular a unas gafas inmersivas para sumergirse en programas de realidad virtual, utilizarlo como interfaz para manejar un gigantesco videomapping, se puede utilizar la pantalla móvil como mando de control de una smart TV, o sencillamente, como disco duro desde el que se lanzan contenidos a la pantalla grande. Por otra parte, estas pantallas móviles buscan una mayor polivalencia de usos. Ya hay tabletas que esconden un proyector capaz de producir imágenes de hasta 50. Así, por ejemplo, es más fácil trabajar en grupo y luego presentar los resultados. 
La convergencia de medios, soportes y equipos se amplía continuamente y con ellos, supuestamente, sus posibilidades expresivas. Precisamente, es en estas nuevas posibilidades expresivas y en la utilidad de las mismas en lo que deberían fijarse los usuarios lectoautores o coautores, pues en muchos casos, estas supuestas innovaciones no lo son tanto y responden a estrategias comerciales dentro de la denominada obsolescencia programada, que, como apunta Lizuka (2007), no solo es tecnológica.

\section{Accesibilidad física, cognitiva, cultural, económica, tecnológica y de contenidos}

Salvo las aplicaciones específicas para móviles, la investigación muestra que el concepto Mobile First + Responsive Web Design está pasando de la teoría a la práctica, pero no es suficiente con utilizar programas como HTML5 que son responsive, pues hay que ir más allá pensando en las tipografías, los contrastes, los metadatos... La accesibilidad cognitiva y cultural presenta las mismas deficiencias que en otras pantallas. Utilizar opciones de "fácil lectura", por ejemplo, es casi una rareza, a pesar que debería ser algo habitual, especialmente en productos educativos. La accesibilidad económica supone una barrera que acrecienta la brecha digital, barrera que, en muchos casos, se suma a la tecnológica, sin olvidar otras como la edad (Butlewski et al., 2015, pp. 24-33).

\section{Resultados. Aproximación taxonómica y creativa al hipermedia móvil}

Con un smartphone u otro dispositivo móvil conectado a internet, mediante una aplicación que aproveche narrativamente las posibilidades tecnológicas de la movilidad, se genera un tipo de hipermedia con "perspectivas, atributos y usos propios" (Siapera, 2012, p. 3), bien diferenciados del hipermedia. Siapera (2012), Logan (2010) y otros recuerdan, siguiendo a McLuhan, que los medios son más que nunca extensiones de las personas. En el hipermedia móvil la metáfora deja de serlo, especialmente en su versiones wearables o vestibles. En 
esta aproximación taxonómica se combinan denominaciones que comienzan a ser habituales con otras, fruto de la investigación cuyo objetivo es vislumbrar las posibilidades presentes y futuras de este nuevo medio. No es difícil inferir sus potencialidades educativas y la importancia de cuidar su accesibilidad física, cognitiva, cultural, económica, tecnológica y de contenidos.

\section{Walking cinema o audiovisual geolocalizado}

Las denominaciones muchas veces se rigen por el marketing y pensando en términos que aparezcan antes y con más frecuencia en las búsquedas de los usuarios en la web, de ahí que no haya que olvidar nunca la actitud crítica. En 2008, Michael Epstein inventa el término walking cinema (Epstein, 2016) para definir el audiovisual geolocalizado. El término cine tiene unas connotaciones más seductoras en el imaginario colectivo que vídeo o audiovisual. Un grupo del MIT Medialab, dirigido por Davenport (2016), que investigaba sobre hipermedia se Ilamaba Interactive Cinema. El walking cinema o audiovisual geolocalizado combina el audiovisual en entornos geolocalizados potenciando la interacción de las narraciones con el espacio, como Murder on Beacon Hill (Figura 3. Walking cinema. Murder on Beacon Hill (https://goo.gl/7SzrBT) que invita a seguir los pasos del profesor de Harvard Dr. John Webster que asesinó a su rico colega el Dr. Parkman porque le reclamaba una deuda. Se hace así un recorrido singular por el Boston del siglo XIX. Aplicaciones como Narratives (Nagler, Hicks et. al., 2014), teóricamente, están pensadas para transformar el relato cinematográfico y sacarlo de la sala de cine, pero, realmente, están orientadas a la producción de audiovisuales interactivos. Estos relatos se viven en el espacio e invitan a viajar en el tiempo. Lemos (2010) afirma que los locative media generan lo que denomina "funciones post mass media" creando una nueva territorialización con nuevos significados del espacio y de la sociabilidad del mismo. 

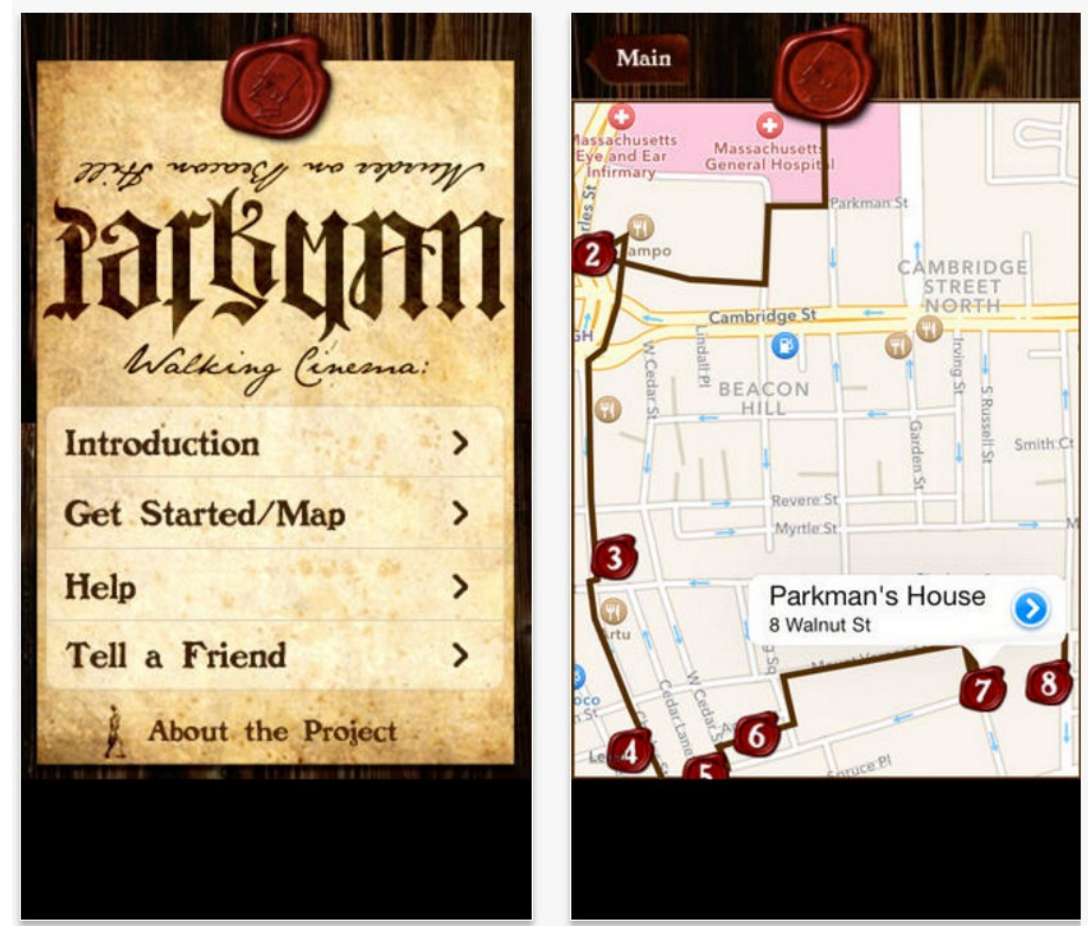

Figura 3 Walking cinema. Murder on Beacon Hill

\section{Hipermedia peripatética}

Crear y reflexionar a la vez que se pasea tiene una larga tradición encabezada por Aristóteles. El hipermedia móvil facilita el proceso de creación tomando datos, reflejando el recorrido, interconectando la creación personal con otras huellas multimedia geolocalizadas, generando así un género que se podría denominar "hipermedia peripatética". Algunas investigaciones focalizan sus trabajos en este sentido, como el Living Mobile del MIT Medialab, dirigido 
por Schmandt (2016), con propuestas como Pintail: A travel companion app for guides storytelling; una ayuda para contar el viaje que se está realizando y crear historias en torno al mismo. La aplicación toma datos directamente para completar la experiencia. El grupo investigador se denominaba anteriormente Speech+Mobility, con una clara intención de investigar el discurso en movilidad.

\section{Realidad aumentada geolocalizada}

La realidad aumentada geolocalizada permite combinar en el entorno real los elementos físicos con los virtuales, o sustituir con los virtuales los reales. Mediante códigos o reconocimiento de objetos, también es posible utilizar un tipo de realidad aumentada multimedia informativa. Aplicaciones como Google Goggles hacen que, a través de la toma de fotografías de cualquier espacio, objeto, imagen... el sistema conecte con las webs que tengan información del mismo. La imagen que se toma es un conector con lo que existe previamente en internet sobre lo encuadrado en la fotografía. Aun con sus muchas deficiencias, estas aplicaciones son de gran utilidad e invitan a profundizar de una manera sencilla sobre cualquier artefacto. La denominada internet de las cosas complementa las tecnologías móviles dando vida a objetos estáticos y proponiendo nuevas formas de involucrarse con lugares, espacios y objetos. Aquí, los dispositivos móviles se convierten en un mecanismo interactivo de visualización y los objetos reales toman vida digital. Un ejemplo es QRator (Hudson-Smith, 2012) que permite a los usuarios crear, recopilar y diseminar información en el espacio del museo.

\section{Walking documentary o documental geolocalizado}

Del walking cinema o audiovisual geolocalizado, como supergénero, se derivan otros géneros y subgéneros. Tal vez el más popular sea el walking documentary o documental geolocalizado. Existen variantes en las que el audiovisual no está geolocalizado sino asociado, por ejemplo, a recorrer un determinado 
número de metros. Así, la aplicación A Walk Through Time (Figura 4. A Walk Trough Time: http://goo.gl/9doAVt) reconstruye un viaje a través del tiempo desde que se forma la Tierra. Cada paso equivale a un millón de años. Hay un acompañamiento sonoro y cuando se alcanzan hitos importantes de la evolución del planeta se visiona un vídeo a la vez que se hace un pequeño descanso de la caminata. Moverse para conocer, sacar el conocimiento a la calle; así lo hace el London Museum mediante la aplicación Street Museum (ver figura 1). La descontextualización que sufre el arte en el museo se palia de esta manera abriendo nuevas posibilidades participativas de educomunicación.

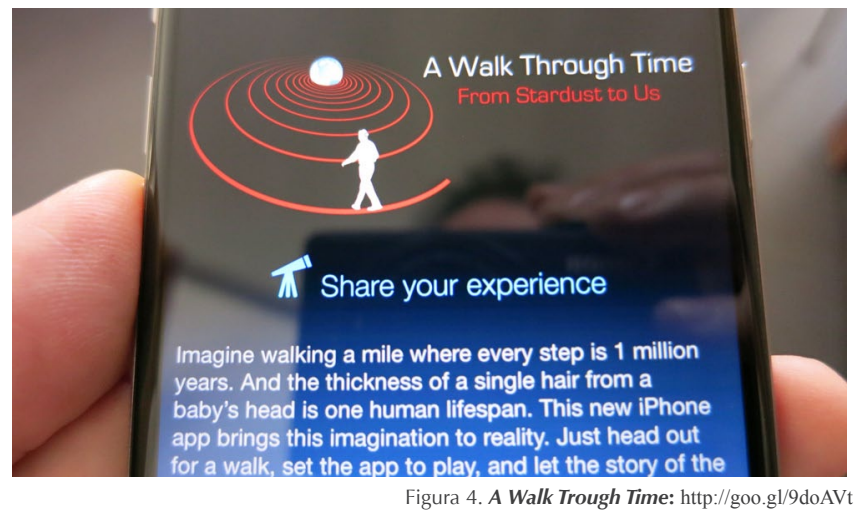

\section{El walking audio o audio geolocalizado}

El audio geolocalizado acentúa las convergencias y las interacciones analógicodigitales. El participante percibe la imagen natural del momento y el audio puede recrear otro tiempo en el mismo espacio. Esa interacción puede ser de seguimiento de un audio móvil significativo que se da en ese espacio solo en fechas concretas como una procesión o un desfile de carnaval. El participante puede seguir, por ejemplo la cabecera del evento para comprender como se desarrolla. Como el audio se graba y geolocaliza en la representación real, 
al hacer el seguimiento en otro tiempo, deberá seguir el mismo ritmo de la representación, sino lo hace así, notará cómo el sonido se aleja o se queda atrás. Es sencillo pensar en otras variantes, como geolocalizar el sonido de la manifestación desde lugares representativos, como el inicio o el final, de manera que el seguimiento se realice deteniéndose en esos lugares para tener una sensación sonora de la evolución del recorrido. Adam y Perales (2013) desarrollaron el proyecto Impronta sónica que consiste en generar un hipotético mapa de América en el parque del Oeste de Madrid frente al Museo de América, por el que es posible pasear para ir descubriendo testimonios de migrantes de los distintos países, enraizándolo con el proyecto Migrar es cultura del mismo museo.

\section{Geocaching hipermedia o búsqueda del tesoro hipermedia}

La tradicional búsqueda del tesoro tiene una vertiente que converge o puede converger con el hipermedia móvil Ilamada geocaching. El geocaching utiliza el GPS para acercarse al objeto escondido (tesoro), pero no lo delimita exactamente para acentuar el sentido lúdico tradicional de dicho juego. Una vez que se descubre el tesoro, se vuelve a esconder y se deja constancia de que se ha encontrado en la web del geocaching. El concepto geocaching hipermedia adquiere su sentido cuando se añaden elementos multimedia al proceso de exploración y al propio objeto. De hecho es habitual añadir, por ejemplo, un chip con información sonora a dicho objeto. Así lo hacen Adam y Perales (2013, p. 127) en proyectos como Derivas virtuales en El Cabanyal. Existen distintas variantes de búsquedas, incluso algunas necesitan aditamentos en el dispositivo móvil, como la lupa microscopio para descubrir en ciertos espacios detalles mínimos que se escapan a la mirada y que el participante deberá fotografiar valiéndose de la misma y subirlos a la web del juego.

\section{Creación artística y aplicada en movilidad}

El hipermedia móvil y los hipergéneros asociados a él propician la creación 
artística redefiniendo los parámetros que se plantean en los proyectos aplicados. Tuters y Varnelis (2006) tachan de nostálgicos a los que critican, desde el arte, que los medios geolocalizados apelan a intereses comerciales y dependen en exceso de una rígida cartografía. Explican cómo estos medios se han distanciado del videoarte y son capaces de escapar de la pantalla y extenderse al ancho mundo. Nickolay Lamn, por ejemplo, se pregunta What if you Could See WiFi y genera una serie de obras tratando de visualizar esa experiencia en paisajes cotidianos. Clara Boj y Diego Díaz, en su obra Observatorio, invitan a descubrir, mediante un dispositivo de realidad aumentada, esas ondas wifi transformadas en representaciones visuales. Esa especie de transliteraciones visuales y sonoras invita a la reflexión sobre ese inquietante mundo invisible que nos rodea. Esther Polak e Ivar Bekkum exploran las intersecciones entre arte y tecnología en su proyecto 250 Miles y manifiestan su pasión por el movimiento. En GPS Museum, desarrollado por Adam y Perales (2016) existe un apartado de locative storytelling en el que se encuentran numerosos ejemplos. La endeble frontera entre el hipermedia móvil aplicado y el de creación artística no suele ser otra que la intención del autor y el género a la hora de realizarlo. Otra cosa bien distinta es que realmente se logre. Sin embargo, esa intención artística suele conllevar una mayor libertad temática, de género, tecnológica... y una decidida búsqueda de la originalidad.

\section{Videojuegos geolocalizados}

En los videojuegos sobre plataformas móviles cada vez se persigue más la interacción narrativa con el espacio y con otras personas acudiendo a distintas convergencias tecnológicas y mediáticas. Por ejemplo, A Robot (Santoso y Gook, 2012) potencia la interacción con el usuario, limitando, sin embargo, las posibilidades narrativas. Viking Ghost Hunt (Naliuka et.al., 2010) utiliza la localización, la orientación y la realidad aumentada para apoyar la mecánica de juego y el relato. En los proyectos de los grupos de los autores se investiga sobre esas convergencias, especialmente entre realidad, realidad aumentada, 
audio y vídeo interactivos geolocalizados. El objetivo es lograr un grado máximo de participación interactiva (constructivo) y de interacción física y personal con el entorno y con otras personas. Prototipos como Hacia Tantalluc (Perea, Moreno et. al. 2015) profundizan en esa dirección.

\section{Hipermedia móvil 360}

Hay que ser conscientes de la juventud del medio y de las limitaciones tecnológicas del mismo. Haciendo inferencias a través del análisis de contenidos y cruzándolos con nuevos desarrollos de hardware y software, en la investigación se plantean numerosos hipotéticos géneros y subgéneros con aplicaciones de muestra. Dada la longitud del artículo, se elige, como ejemplo, un solo género de inmersión interactiva audiovisual en el que las interacciones sean similares a las de la vida real: el que se podría calificar de hipermedia móvil 360. Por más puntos de vista que incorpore la ventana audiovisual, incluso utilizando objetivos ojo de pez $\left(180^{\circ}\right)$, siempre plantean interactividades e interacciones limitadas; sin embargo, utilizando cámaras de $360^{\circ}$, la persona que participa puede elegir el punto de vista como lo hace en la realidad. Con un software adecuado y con unas gafas especiales de realidad virtual, la interactividad e interacción audiovisual puede ser total en cualquier recorrido. Se alcanza así a una especie de realidad virtual de la realidad. Si a eso se uniese que la cámara 360 grabase en 8K (7680×4320, frente a la HD actual de (1920x1080), realidad y virtualidad solo se diferenciarían por las condiciones meteorológicas. A las $8 \mathrm{~K}$ se les denomina tecnología terminal, pues, aunque se aumentase aún más la definición, el ojo no tiene capacidad para apreciarlo. Puede pensarse que no es necesaria tanta definición para una pantalla tan minúscula como las de unas gafas. Para planos generales, no; pero es imprescindible para llegar al detalle deseado sin perder realismo.

Si se reflexiona sobre el grado de participación en ese hipotético hipermedia móvil 360, se observa que es selectivo; hacerlo transformativo y constructivo 
plantea retos aún mayores a la tecnología. Piénsese en la complejidad de intervenir sobre el contenido narrativo documental (personajes, espacio, tiempo y acciones) y en su manifestación discursiva (imágenes audiovisuales, fotográficas, infográficas y tipográficas). No es descabellado pensar que todos los elementos reales pudieran convertirse en infográficos tridimensionales $y$, por tanto, en elementos manipulables por el usuario coautor. Aunque la mercadotecnia busque persuadir de lo contrario, intentando deslumbrar en lugar de alumbrar el conocimiento; la imaginación va siempre por delante de la tecnología y ésta debe procurar estar a su servicio materializando esas intuiciones.

\section{Hipermedia móvil en los procesos transmedia}

Es difícil concebir un proceso transmedia sin que estén presentes distintas variantes del hipermedia móvil. Es más, éstas suelen erigirse en protagonistas (Moreno y Navarro, 2015). La pregunta es si realmente pueden considerarse transmedia muchos proyectos o simplemente son catálogos de medios utilizando un mismo contenido. La creación transmedia exige partir de un mismo contenido y utilizar distintos medios para su difusión aprovechando las características narrativas de cada uno de ellos que mejor se adaptan a las distintas partes del contenido. Y lo más importante, que contenidos y discurso de todos los medios utilizados sean complementarios, formando así un conjunto que se comprende y disfruta cuando se participa de la totalidad. Transmedia requiere una estrategia narrativa completa y no una mera estrategia comercial que suma medios sin tener en cuenta las interacciones entre los mismos y una imprescindible participación de las personas convertidas en coautoras.

\section{Conclusiones}

La posibilidad de crear relatos multimedia interactivos o participar en ellos sin limitaciones espaciales o temporales, interactuando e interaccionando 
con el entorno en movilidad genera un nuevo medio. Las tecnologías móviles en convergencia con la geolocalización y otras tecnologías que facilitan interacciones mimético-naturales lo hacen posible. Cualquier persona con el dispositivo adecuado es un coautor. Lectura, cocreación y creación hipermedia se aúnan en un mismo proceso de experimentación narrativa diferente y novedosa que enriquece la memoria individual y colectiva (Ladly, Buzio y Ludlow, 2014). Conviene destacar que varias de las herramientas que potencian el desarrollo de aplicaciones hipermedia e hipermedia móvil son de software y hardware abierto (Cangiano y Fornari, 2014). Se apunta así al surgimiento de paradigmas de desarrollo en donde la personalización se une a la fabricación personal, al desarrollo de productos y al codiseño por parte de los usuarios. Estos síntomas le llevan a Rifkin (2014) a pensar en una futura sociedad de coste marginal cero.

El desarrollo de la narrativa hipermedia móvil requiere un trabajo inter y transdisciplinar capaz de integrar todo tipo de elementos tecnonarrativos para la creación de relatos en los que la tecnología resulte invisible. Las complejas capas tecnológicas deben constituir la parte oculta del iceberg para que las personas disfruten de los contenidos, la parte visible del mismo. Ello requiere un conocimiento profundo de la narrativa y de la tecnología para Ilegar una transmedialidad en la que se inserte el hipermedia móvil. Convergen aquí los múltiples sensores y mecanismos del actual internet de las cosas, todo tipo de dispositivos móviles y sus aditamentos, conocimientos profundos de infografía, computación gráfica, inteligencia artificial y la conjunción de teorías matemáticas, humanísticas y de comunicación para alcanzar interactividades e interacciones al servicio de las personas. Solo así la lectoautoría puede hacerse realidad de una forma sencilla, accesible y amigable. La accesibilidad física, económica, cognitiva, cultural, tecnológica y de contenidos debe guiar los procesos creativos y productivos. La accesibilidad es precisamente una de las mayores deficiencias de este nuevo medio en formación. Deficiencias que deben potenciar una actitud crítica hacia un mercado que no es capaz 
de conjugar los beneficios para las personas con el razonable beneficio económico, sino que privilegia este último a través de la tecnología de la obsolescencia programada que deslumbra y ensombrece los contenidos.

Sin olvidar esa actitud crítica, hay que mirar los dispositivos móviles, especialmente los smartphones, como un apoyo creativo y ludoeducomunicativo en lugar de una amenaza. Ese es el gran reto que se les plantea a todas las personas, pero especialmente a la academia. Nativas y nativos digitales lo tienen muy claro, el móvil es ya una extensión de su cuerpo. Tratar de eliminarla de los espacios educativos constituye una quimera. Contar con ella, una ventaja educativa y creativa.

\section{Referencias}

Adam, F. y Perales, V. (2016). GPS Museum (http://goo.gl/9iyTQL).

. (2013). Integración de GIS (sistemas de georreferenciación de la información) y localización espacial en prácticas pedagógicas y lúdicas vinculadas a museos. Arte, individuo y sociedad, v. (25), 121-134 (http://goo. gl/h74Qel).

Beranuy, C. et al. (2009). Validación de dos escalas breves para evaluar la adicción a Internet y el abuso de móvil. Psicothema,v. 21, núm. 3, 480-485 (http://goo.gl//Kf4HT).

Bush, V. (1945). "As We May Think". The Atlantic, July 1, 1-8. (http://goo.gl/ (kfceA).

Butlewski, M. et al. (2014). Heuristics in Ergonomic Design of Portable Control Devices for the Elderley. En: Stephanidis, C. y Antona, M. (Eds). Universal Access in Human-Computer Interaction: Aging an Assistive Environment. Heidelberg: Springer. 
Calvi, J. (2010). La reconfiguración del campo cultural. Lógicas sociales de creación y circulación del audiovisual en Internet. Telos, 85, 45-52. (http:// goo.gl/RP7jcr).

Cangiano, S. y Fornari, D. (2014). Products as platforms: a framework for designing open source interactive artifacts. En: Proceedings of the 2014 Companion Publication on Designing interactive systems (DIS Companion '14),219-222. (http://goo.gl/CgxmgX)

Davenport, G. (2015). Interactive cinema. MIT Medialab (http://goo.gl/OHkSqt).

Ditrendia (2015) (http://goo.gl/BD8Zun) .

Epstein, M. (2016). Walking cinema. En: Museum I+D+C (2016). Glosario Social Expandido (https://goo.gl/rsQ0Ks).

Gómez Alzate, A. (2015). Modelo de Diseño para la valoración y apropiación social del Patrimonio en el Paisaje Cultural Cafetero Colombiano. Kepes, año 11, núm. 11 enero-junio, 117-138. DOI: 10.17151/kepes.

Grassi, A., Gagglioli, A. y Riva, G. (2009). The green valley: the use of mobile narratives for reducing stress in commuters. Cyberspsychology. Behavior and Social Networking, April, num. 12, v. 2, 155-161. (http://goo.gl/j6GNvA).

Hudson-Smith, A. et. al. (2012). Experiments with the internet of things in museum space: QRator. En: Proceedings of the 2012 ACM Conference on Ubiquitous Computing (UbiComp '12),1183-1184. (http://goo.gl/MZzzyK).

Lizuka, T. (2007). An Empirical Analysis of Planned Obsolescense. Journal of Exonomics \& Management Strategy, January, v. 16, num. 1, 191-226. (http:// goo.gl/PzFBAH).

Ladly, M.; Ludlow, B. y Buzio, G. (2014). People, places and things: a mobile locative mapping workshops. En: Proceedings of the 16th international conference on Human-computer interaction with mobile devices \& services (MobileHCl '14),569-572 (http://goo.gl/3ea7Mq). 
Landow, G. P. (2006). Hypertext 3.0. Critical Theory and New Media in an Era of Globalization. Maryland, USA: The Johns Hopkins University Press.

Lemos, A. (2010). "Post-Mass Media Functions, Locative Media, and Informational Territories: New Ways of Thinking About Territory, Place, and Mobility in Contemporary Society". Space and Culture, November, v. 13, núm. 4, 403-420. (http://goo.gl/3ea7Mq).

Logan, R. K. (2010). Understanding New Media: Extending Marshall McLuhan. New York: Peter Lang Publishing.

Moreno Sánchez, I. (2015). Interactividad, interacción y accesibilidad en el museo transmedia. Zer, Vol. 20, núm. 38, 87-107. (http://goo.gl/yVAsUA).

Moreno Sánchez, I. y Navarro Newball, A. A. (2015). La ciudad escondida. Toledo, laboratorio de comunicación transmedia. Opción, año 31, núm. 1, 806-827 (http://goo.gl/ayOPZB).

Moreno Sánchez, I y Navarro Newball, A. A. (2013). Comunicación cultural y TIC: La representación accesible de la cultura Chimú. Historia y Comunicación Social. Vol. 18, octubre, pp. 541-554. DOI: http://dx.doi.org/10.5209/rev_ HICS.2013.v18.43987

Nagler, S.; Hicks, A. et. al. (2014). Narratives: geolocative cinema application. En: Proceedings of the 16th international conference on Human-computer interaction with mobile devices \& services (MobileHCl '14), 593-595. (http:// goo.gl/JF92LD).

Perea Tanaka, C. Moreno Sánchez, I. et. al. (2015). Towards tantalluc: interactive mobile augmented reality application for the Museo de América de Madrid. Computing Colombia Conference, IEEE Xplore (http://goo.gl/K8fVVi).

Rico, J. (2010). Usable Gestures for Mobile Interfaces: Evaluating Social Acceptability. En: CHI'10 Proceedings of the SIGCHI Conference on Human Factors in Computing Systems, 887-896(http://goo.gl/Arh3iz). 
Rifkin, J. (2014). The Zero Marginal Cost Society: The Internet of Things, the Collaborative Commons, and the Eclipse of Capitalism. New York: Palgrave Macmillan.

Santoso, M. y Gook, L. B. (2012). ARobot: development of 3rd person shooting game and handheld augmented reality. En: Proceedings of the 11th ACM SIGGRAPH International Conference on Virtual-Reality Continuum and its Applications in Industry (VRCAI '12),55-58. (http://goo.gl/Kw6pFR) (23-02-2016).

Schmandt, C. (2016). Living Mobile (http://goo.gl/tHt5pC).

Siapera, E. (2012). Undestanding New Media. Londres: SAGE.

Townsend, A. (2006). Locative-media Artists in the Contested-Aware City". Leonardo, v. 39, num. 4, 345-347(http://goo.gl/3ZwOSk).

Tuters, M. y Varnelis, K. (2006). Beyond Locative Media: Giving Shape to the Internet Thing. Leonardo, August, v. 39, núm. 4, 357-363.

Cómo citar este artículo:

Moreno, I. \& Navarro, A.A. (2016). El hipermedia móvil como nuevo medio y su protagonismo en la creación transmedia. Revista Kepes, 13, 145-170. DOI: 10.17151/kepes.2016.13.13.8 